\title{
Health Communication: A Key Component of Public Health
}

Health communication is the science of understanding how health information is generated and disseminated and how that information affects various beneficiaries as listed in Figure 1. The field includes the study of secular communication, as well as the strategic communication of evidence-based health information to professional and nonprofessional audiences.

Facts about health and behaviors are shaped by the communication, information and technology that people interrelate everyday. Health communication and health information technology (IT) are central to healthcare, public health and the way our culture views health. These processes make up the framework and also the ways professionals and the public search for, and use health information considerably impacting their health decisions and actions. Communication programs can have multiple areas of expansion as listed in Figure 2.

Patients' perceptions about the quality of the healthcare they received are highly dependent on the quality of their interactions with their healthcare clinician and team. ${ }^{1,2}$ There is a wealth of research data that supports the benefits of effective communication and health outcomes for patients and healthcare teams. Greater support is also needed for research and evaluation in public health communication, i.e. truly transdisciplinary simultaneously addressing

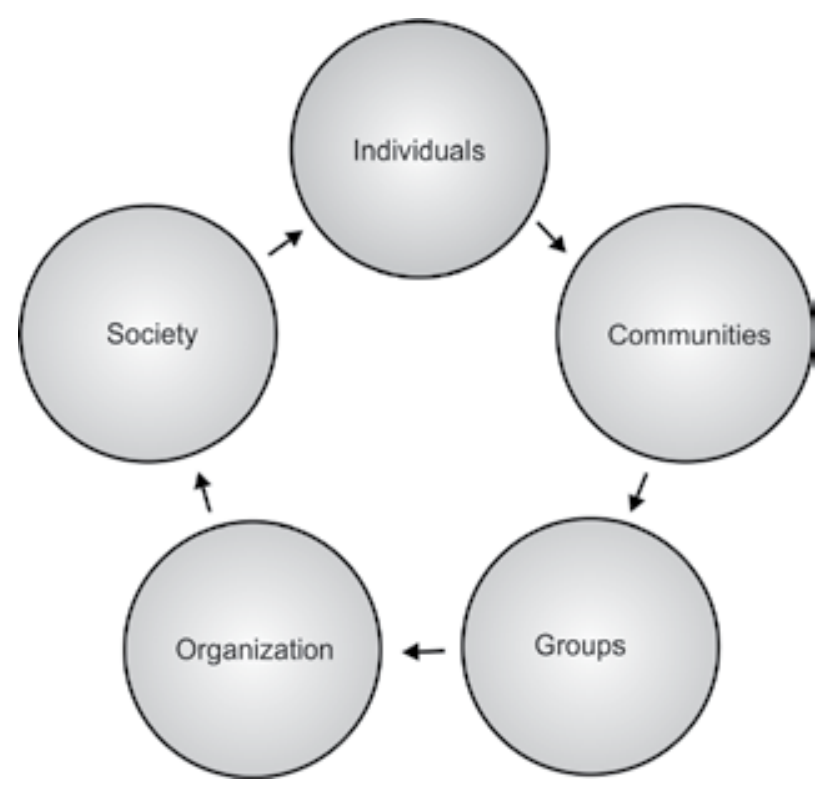

Fig. 1: Health communication beneficiaries

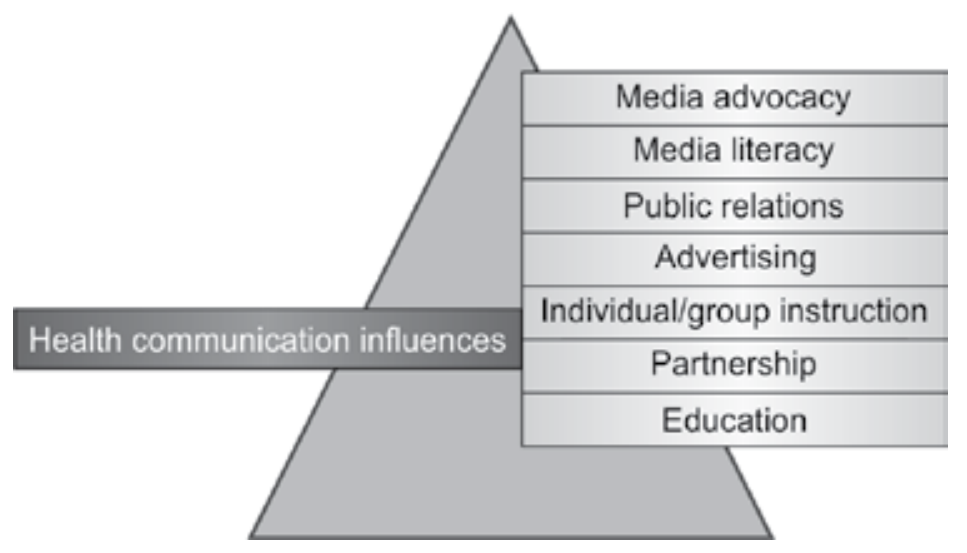

Fig. 2: Effective health communication components 
multiple health issues, intervention levels and communication channels. Further, although there are many challenges for program evaluation in public health communication; conducting comprehensive evaluations and disseminating the results is critical for expanding knowledge, improving programs, and allocating limited resources. ${ }^{3}$ Public health communication recognizes that for programs to be both ethical and effective, the intended audience should be informed about all stages of an interventions including development, planning and implementation to ensure that the program reflects the audience's ideas, needs, and values. ${ }^{4,5}$

Health communication initiatives must use the most effective and efficient strategies for the promotion, protection and maintenance of health through the use of the best available evidence at practice and policy level. Public health practitioners, program managers and policymakers need to be aware about the strengths, weaknesses and costs of health communication interventions aimed for the prevention and control of communicable diseases so that impacts can be enhanced and opportunities can be maximized further strengthening the evidence-informed action.

The dynamic health communication between policymakers, health providers, clinicians, researchers and public health institutions is operational at all levels of health promotion from the grassroots community level all the way to the international establishments. When patient care is harmonized between medical and dental professionals, the result is a higher level of care, fewer impediments, and abridged costs for both patient and physician. This will also able one to understand the pulsating relationship between oral-systemic health especially among physician, dentists and public.

\section{REFERENCES}

1. Clark PA. Medical practices 'sensitivity to patients' needs: opportunities and practices for improvement. J Ambul Care Manage 2003 Apr-Jun;26(2):110-123.

2. Wanzer MB, Booth-Butterfield M, Gruber M. Perceptions of health care providers' communication: relationships between patientcentered communication and satisfaction. Health Commun 2004;16(3):363-384.

3. Bernhardt JM. Communication at the core of effective public health. Am J Public Health 2004 Dec;94(12):2051-2053.

4. Institute of Medicine. Speaking of Health: Assessing Health Communication Strategies for Diverse Populations. Washington, DC: The National Academies Press; 2002.

5. Guttman N. Public Health Communication Interventions: Values and Ethical Dilemmas. Thousand Oaks (CA): Sage Publications; 2000.

Editor-in-Chief

Rajiv Saini

Associate Professor

Department of Periodontology and Oral Implantology

Pravara Institute of Medical Sciences

Loni, Maharashtra, India 